\title{
COUPLED THERMO-ELECTRO-MECHANICAL MODELS OF CARDIAC ABLATION AT TISSUE-CELLULAR SCALES AND A ROLE OF MICROTUBULES
}

\author{
SUNDEEP SINGH ${ }^{1}$ and RODERICK MELNIK ${ }^{1,2}$ \\ ${ }^{1}$ MS2Discovery Interdisciplinary Research Institute, Wilfrid Laurier University, \\ 75 University Avenue West, Waterloo, Ontario, N2L 3C5, Canada \\ rmelnik@wlu.ca, http://www.m3ai.wlu.ca/ \\ 2 BCAM, Alameda de Mazarredo 14, E-48009 Bilbao, Spain
}

Key words: Radiofrequency Ablation, Cardiac Ablation, Thermo-electro-mechanical Coupling, Bioheat Transfer, Hemodynamics, Fluid-structure Interaction, Cytoskeleton and Microtubules, Modelling

\begin{abstract}
Radiofrequency ablation is a medical procedure that is becoming increasingly used for disease treatments. During this procedure, part of dysfunctional tissues is ablated by using the heat, typically generated from medium frequency electric current. It is a field of medicine where mathematical and computational models play a substantial role in assisting clinical practitioners with quantifications of some of the most critical characteristics, including temperature distributions and ablated volumes. In this contribution, we describe a framework for the development of coupled thermo-electro-mechanical models in this field. While our framework and the described validation procedures can be applicable to a variety of ablation modalities and treatments, a major focus has been given to some of the pecularities related to cardiac ablation at tissue-cellular scales and a role played by cell organelles such as microtubules, as well as by the cell nucleus. We have discussed the effects their inclusion makes on the calculation of the main characteristics of the radiofrequency ablation procedures. The importance of domain heterogeneity, as well as the integration of fluid-structure interaction in the developed framework along with other effects, have been highlighted and the details on ablation modalities in the context of clinical experimental research have been given. Finally, future generalizations of the proposed framework with hybrid stochastic-deterministic models have been put forward.
\end{abstract}

\section{INTRODUCTION}

The application of radiofrequency ablation (RFA) has been widely explored in treating various types of diseases and pain, including cardiac arrhythmias. Computational modelling provides a safe and viable alternative to ex vivo and in vivo experimental studies for quantifying the effects of different variables efficiently and reliably, in addition to providing a priori estimates of the ablation volume attained during such procedures.

In a quest for minimizing the deviations between the numerically predicted and experimentally obtained ablation volumes during the RFA procedures, different strategies have been explored. In this contribution, we report a fully coupled thermo-electro-mechanical model [1] for a more accurate prediction of the RFA treatment outcomes. While the developed modelling framework is applicable to a range of problems in RFA, a major focus here has been given to cardiac ablation procedures. This has been motivated by the 
fact that minimally invasive radiofrequency cardiac ablation (RFCA) has emerged as one of the most promising and efficient modalities for treating certain types of cardiac arrhythmias. Around 35 million people worldwide are affected by various forms of arrhythmias, with $2 \%$ to $3 \%$ of the population in Europe and North America. It is a common cardiac disease related to abnormal heart rhythms that can cause symptoms like heart palpitations, shortness of breath, and low energy.

During RFCA, a catheter is navigated into the heart to locally heat and destroy a small tissue region, to electrically isolate the regions that generate or propagate arrhythmia and makes it inactive. Nowadays, computational modeling and simulations have become a significant player in the design and development of new systems along with the improvement and optimization of the existing protocols of RFCA [2].

A three-dimensional model comprising of cardiac tissue and cardiac chamber has been developed in which an electrode is inserted perpendicular to the cardiac tissue to simulate actual clinical procedures $[3,4]$. Temperature-dependent specific heat, electrical and thermal conductivities, along with damage dependent blood perfusion rate have been considered to model a more realistic scenario. The effects of intramyocardial capillary blood flow, elastic moduli and Poisson's ratios on the efficacy of such a fully coupled model of RFA has been systematically investigated. A multiscale model of cardiac tissues has also been developed in the context of quantification of the RFA effects at the cellular level with a particular focus on microtubules [5], modelling of which has been discussed along with other organelles.

The rest of the paper is structured as follows. First, we describe the core modelling components, essential for the development of coupled models in this field. Then, we focus on the importance of domain heterogeneity, providing insight into the coupling of macroscale models at the tissue level and microscale models at the cellular level to the medical procedurtes the context of TTFields other anchoring organel having cardiac ablation in mind,
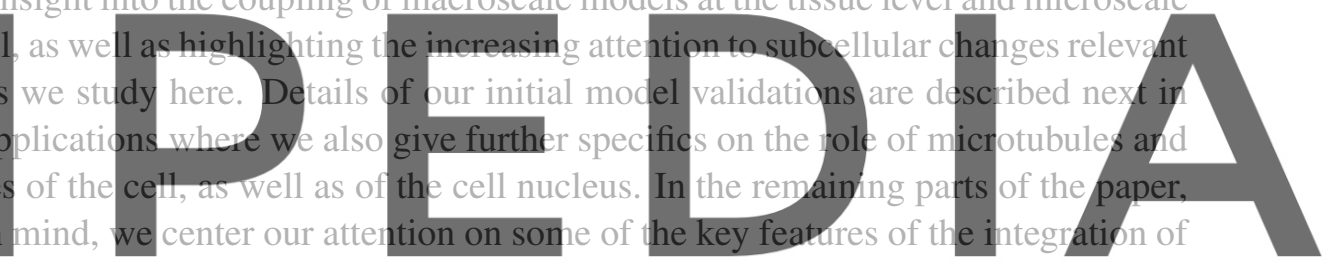

fluid-structure interaction and incorporating other effects in the developed framework. This is followed

\section{Register foy the discussipe on allation. modalities related exparimental studies and conclusionthout the watermark}

\section{THE CORE EQUATIONS AND FURTHER DEVELOPMENTS}

The starting point of the model development includes the perfusion-based biological heat transfer equation $[1,6,7]$ :

$$
\rho_{t} c_{t} \frac{\partial T}{\partial t}=\nabla\left(k_{t} \nabla T\right)-\omega_{b} \rho_{b} c_{b}\left(T-T_{b}\right)+Q_{m}+Q_{p}
$$

where the four terms in the right hand side part accounts for the heat conduction, blood perfusion, metabolism, and electric power, respectively, with subindeces $t$ and $b$ indicating tissue and blood properties. For example, the last term is responsible for the volumetric heat generation within the biological tissue during the thermal ablative procedure and is given by $Q_{p}=\sigma|E|^{2}$. The equation (1) is coupled with a simplified version of the Maxwell equations, used in the quasi-static approximation:

$$
\nabla \cdot[\sigma+i \omega \varepsilon \nabla V]=0, \quad E=-\nabla V, \quad J=\sigma \cdot E,
$$

where the main notations used in this coupled system are: $\mathrm{T}$ is the temperature, $\mathrm{E}$ is the electric field, $\mathrm{J}$ is the current density, $\sigma$ is the electric conductivity, $\varepsilon$ is the relative permittivity, $\omega$ is the angular fre- 
quency, $\rho$ is the denisty, $\mathrm{k}$ is the thermal conductivity, $\omega_{b}$ is the blood perfusion rate, and $c$ is the specific heat capacity. In the context of cardiac ablation, the myocardium tissue has been modeled as a nearly incompressible, isotropic and homogenous hyperelastic material, with the Green strain energy function described by the Mooney-Rivlin model, so the above system was suppelement by the thermoeleastic part describing wave propagation applicable in the case of nonrigid mechanics:

$$
\rho \frac{\partial^{2} u}{\partial t^{2}}=\bar{\sigma}_{i j, j}+F_{i}, \quad \bar{\sigma}=J^{-1} \frac{\partial W(F)}{\partial F} F^{T}
$$

where $\bar{\sigma}$ is the Cauchy stress tensor for the hyperelastic material, $J$ is the total volume ratio, and $W$ describes Green's strain components as mentioned above, with further details given in the above cited papers.

The first analysis of dynamic changes in cells subjected to pulsed RFA, accounting for microtubules, was presented in [8]. While pulsed radiofrequency (PRF) treatments are less destructive compared to their continuous counterparts, they can still adversely affect microtubules and microfilaments of the cells both (i) directly, acting by PRF electric fields on their charged structures and (ii) indirectly, causing damage to the mitochondria and degrading the microtubules and microfilaments. Within the developed framework, this phenomenon can be assessed quantitatively, which is quite important for medical applications of RFA.

\subsection{Domain heterogeneity and tissue-cellular scales}

The models for RFA are multiscale as they couple macroscale models at the tissue level and microscale models at the cellular level. In its turn, each of these
at the tissue level, we couple together the thermal
and electromagnetic models for predicting (a) tem
deformation, (c) electric field and resistive heatin
single-cell knowledge, we may need to include sul
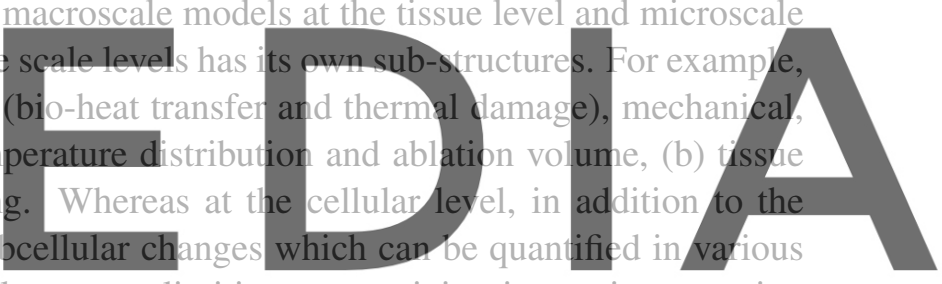

cell types such as neurons and microglia. These latter peculiarities are receiving increasing attention

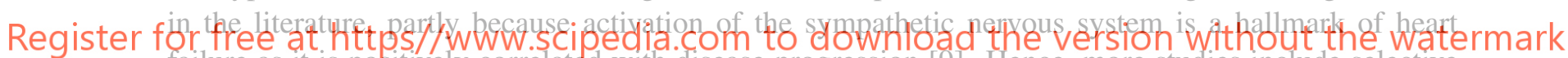
failure as it is positively correlated with disease progression [9]. Hence, more studies include selective

ablation of neurons and measurements of cardiac autonomic tone, function, and arrhythmia incidence, demonstrating an important role of such neurons in cardiac autonomic imbalance, arrhythmogenesis and cardiac dysfunction. Furthermore, the complexity of clinical cardiac syndromes of heart failure, that affect dozens of million people worldwide, requires also a closer look at cellular and molecular levels of neuroinflammation in heart failure which may even have to include brain regions involved in sympathetic control [10]. Notably, in the context of RFA, nerve locations with respect of other tissues may play a critical role [11], and the nerve tissue response deserves a separate detailed analysis [12]. The above considerations made it clear that we deal with problems which are highly heterogeneous at the patient, tissue, cellular, and molecular levels, and this multiscale heterogeneity poses significant challenges for the development of effective therapies, and hence for computational modelling. This issue has been recently addressed in various contexts of RFA in $[13,14]$, where it was demonstrated that the consideration of heterogeneity within the computational domain results in distorted electric field distribution and leads to a significant reduction in the attained ablation volume during some of RFA applications. Further details have been provided in a recent review paper on thermal ablation of biological tissues [7]. 


\subsection{Role of microtubules and other anchoring organelles of the cell}

The importance of cellular scales, highlighted in the previous section, makes us look into additional details at the cytoskeleton of a cell which provides its support through a sophisticated network of protein fibers supporting cell shape and anchoring organelles within the cell. Among them there are three main structural components: (a) microtubules (MTs), formed by tubulins, (b) microfilaments, formed by actins, and (c) intermediate filaments. Recently, the effect of mechanical degradation of the cytoskeleton on the electro-elastic response of the cell has been analyzed in [15] where a general framework has been developed for more accurate quantification of the mechanical/electrical transduction within the biological cells that can be critical for capturing the complex mechanisms at cellular length scales.

These studies are important in the context of the cardiac MT networks which regulate contractile amplitudes and kinetics by acting as a cytoskeletal shock-absorber [16]. However, given that MTs provide breakable cross-links in the cytoskeleton, resisting rapid length changes during shortening/stretching and that MTs increase myocyte viscoelasticity, these findings also suggest that further developments of our models may be necessary to account for a viscoelastic mechanism. The role of MTs and associated mechanisms of viscoelasticity in the context of therapeutically intractable features of heart failure have recently been confirmed by experimental studies as well [17]. As we pointed out in the previous section, a subcellular level may also be necessary to be accounted for, because of the regulatory properties of MTs and MT-associated proteins which are critical for the correct function of a range of tissues. Indeed, there is increasing evidence to suggest that perturbations of the tubulin code can be linked to various diseases, including neurodegenerative, whereas the tubulin code can provide the key to our better understanding

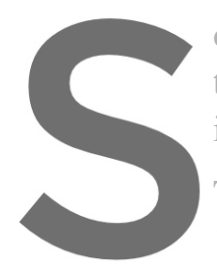
of MT mechanical and dy the functional roles and $\mathrm{n}$ in [19].

The initial testing of the modelling framework was 1a). The results obtained (c.g., Fi
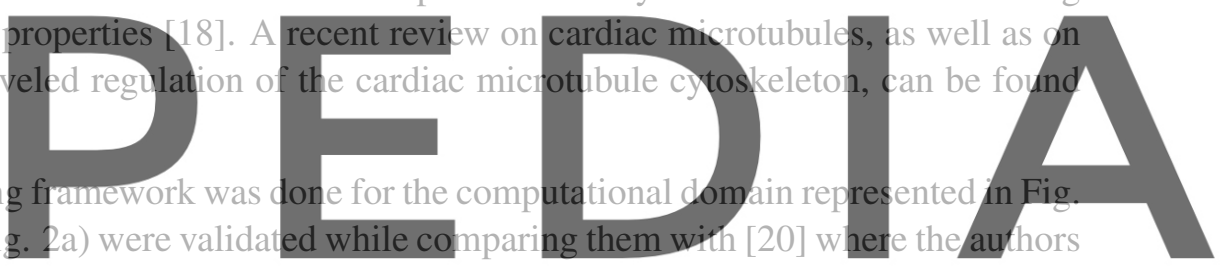

considered a single cell model in the context of TTFields applications. Further results were obtained in

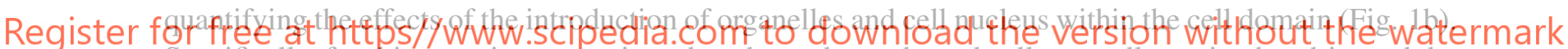
Specifically, for this experiment, we introduced membrane-bound cell organelles, mitochondria, and the results of comparisons of the electric field distributions with and without these additions can be seen in

Fig. 3. Our subsequent analysis included organelles and the cell nucleus as described above. Given that our initial validation of the developed coupled model was done in the context of TTFields applications, a few additional representative examples included the analysis of the effect of increasing frequencies on the electric field which is typical in that context (see Fig. 4). Among other characteristics analyzed during the validation process, we present here electric potential distributions for different applied field strengths (Fig. 5) and the dynamics of temperature evolution within the cell exposed to RF procedures (Fig. 6).

Having in mind a generalization of the computational domain represented schematically in Fig. 2b, further analysis has been carried out. By using a coupled model, the electro-mechanical behavior of microtubules has recently been systematically investigated based on a continuum-based finite element framework [21]. The results suggest that the electro-elastic response of microtubules is significantly dependent on both the magnitude and direction of the applied forces, whereas the output potential is linearly proportional to the predicted displacement and the electric potential within the microtubule. 


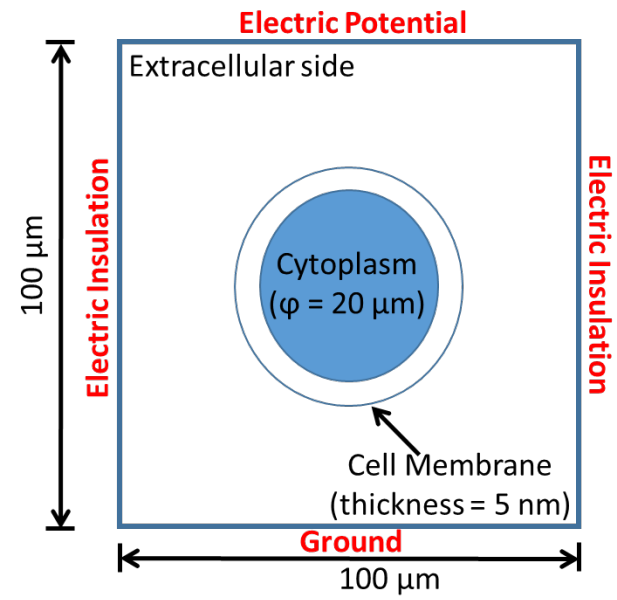

(a)

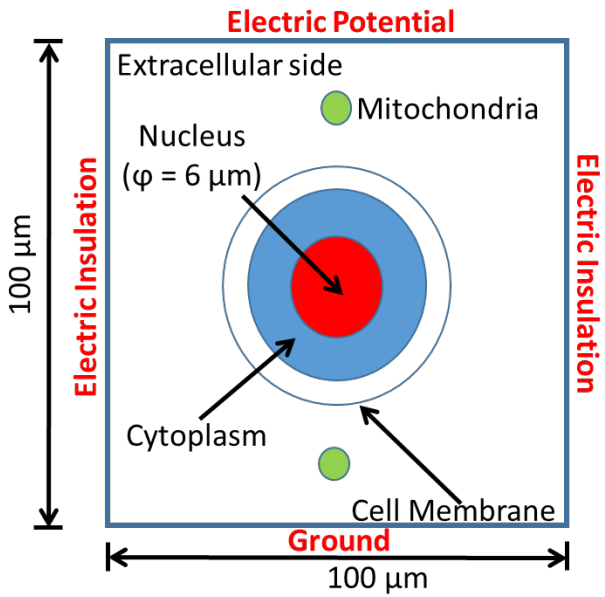

(b)

Figure 1: Computational domain related to the model validation: (a) without organelles/nucleus and (b) with mitochondria organelles and nucleus in place.
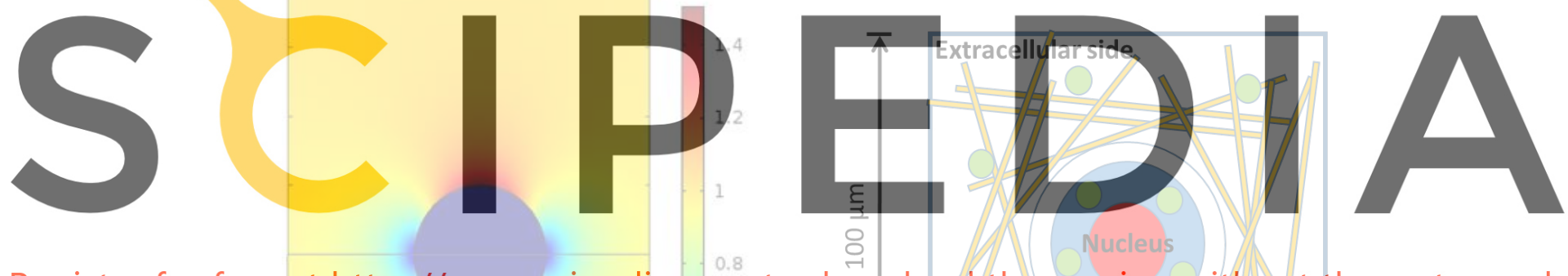

Register for free at https//www.scipedia.com
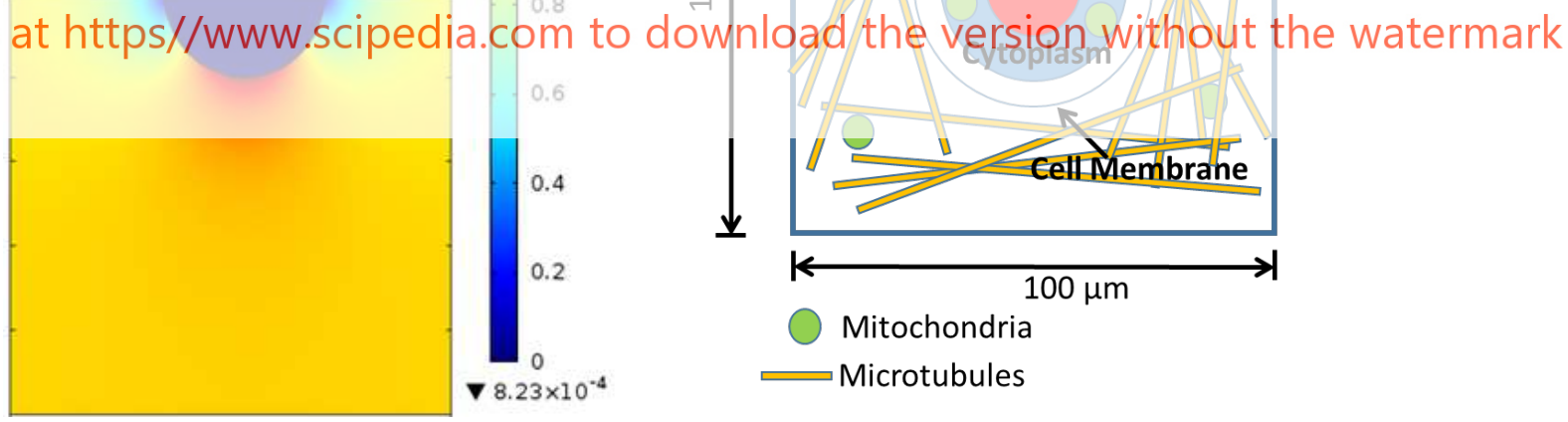

(a)

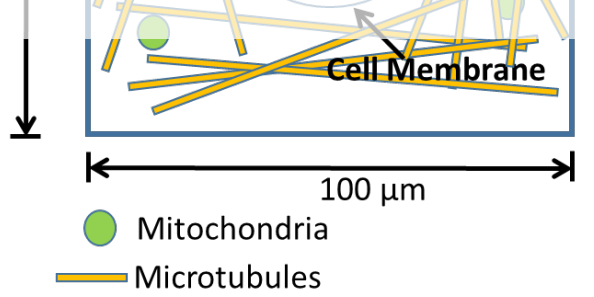

(b)

Figure 2: (a) Results of the test for the electric field distribution at $60 \mathrm{~Hz}$. (b) Schematic of the computational domain with mitochondria/nucleus and microtubules in place.

Further, the effect of mechanical degradation of microtubules on their electro-elastic response has been quantified in [22] and a more complete analysis that includes microtubules has been reported in [15]. These studies provide useful insight on the role of microtubules in different applications of biomedical 


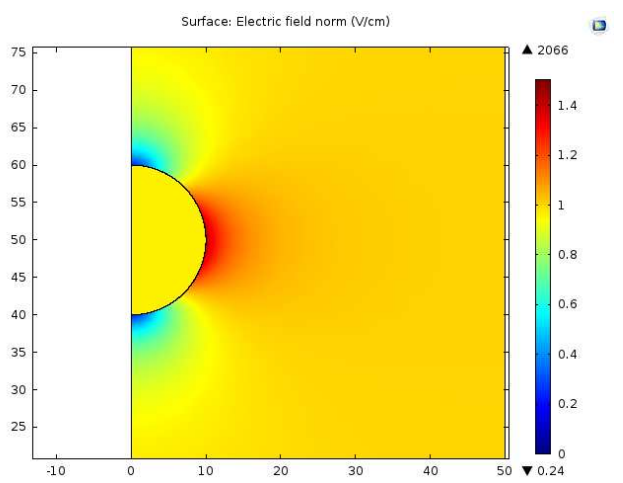

(a)

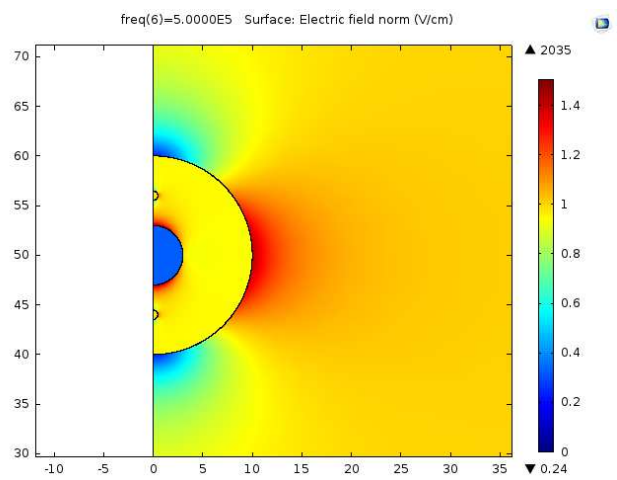

(b)

Figure 3: Electric field distrubutions (a) without organelles (mitochondria) and cell nucleus and (b) with them included into the computational domain. Results are pesented for $500 \mathrm{kHz}$.

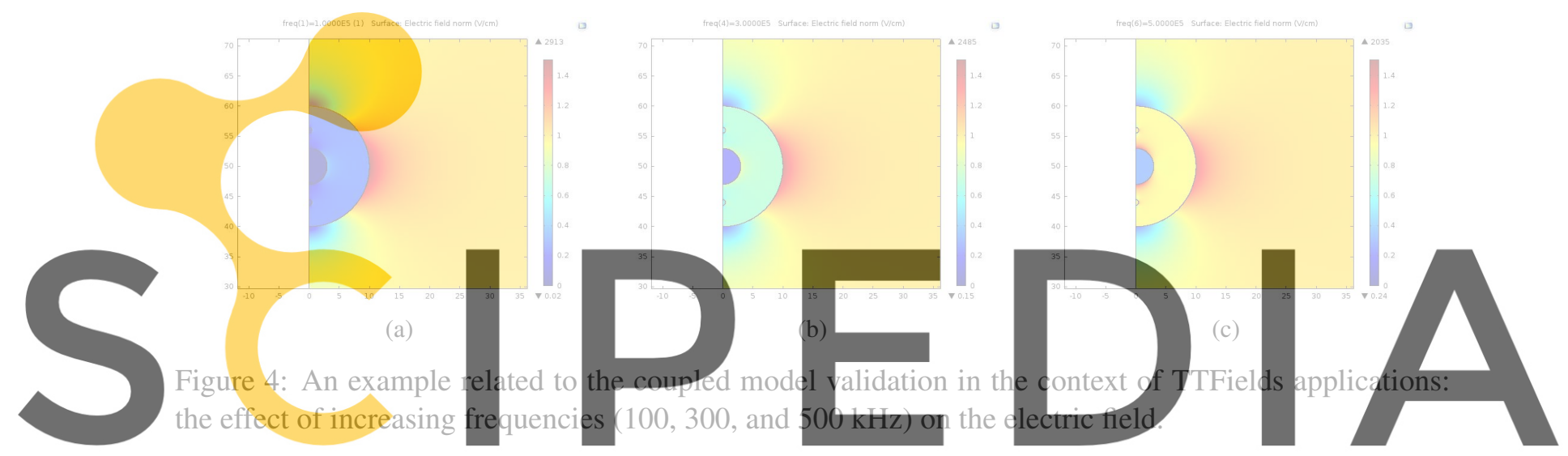

Register for free at https//WwWw.Scipedia.com to download the version without the watermark

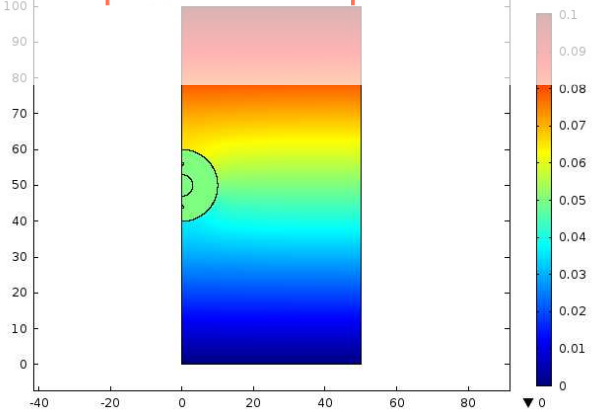

(a)

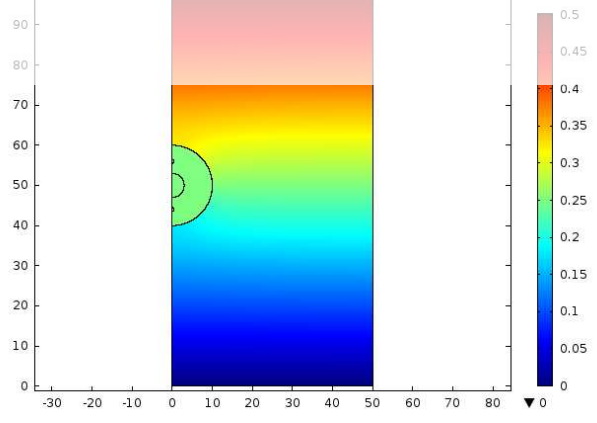

(b)

Figure 5: Electric potential distrubutions for different values of applied field strength at $500 \mathrm{kHz}$, presented for $10 \mathrm{~V} / \mathrm{cm}$ (a) and $50 \mathrm{v} / \mathrm{cm}(\mathrm{b})$.

engineering, such as biocompatible nano-biosensors for health monitoring, drug delivery, as well as 


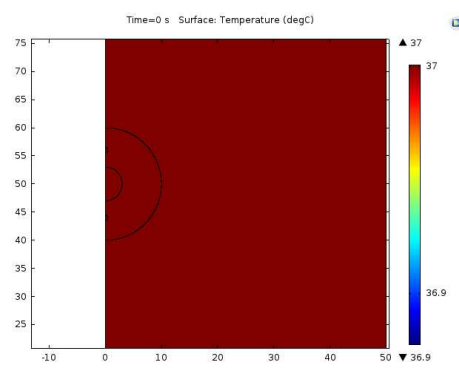

(a)

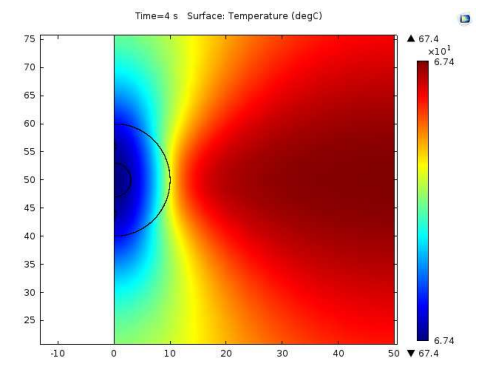

(b)

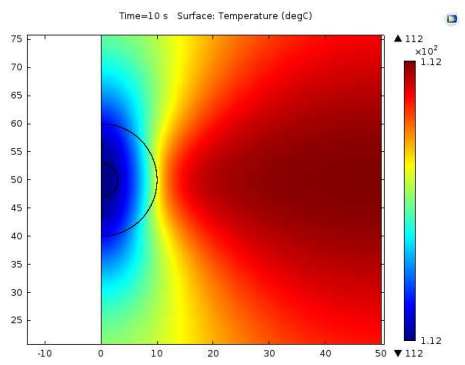

(c)

Figure 6: Dynamics of temperature evolution within the cell exposed to $500 \mathrm{kHz} \mathrm{RF}$, presented for the initial condition (a), at $4 \mathrm{~s}(\mathrm{~b})$, and at $10 \mathrm{~s}$ (c) after the application of the procedure.

noninvasive diagnosis and treatments, including cardiac ablation.

\subsection{Integration of fluid-structure interaction and incorporating other effects}

Further developments discussed here can also include more refined nonlinear models to account for additional features of thermo-electro-mechanical effects in cardiac tissues such as different thermo-electric and nonlinear conductivity properties [23]. In particular, this can be useful for further advancing the models with RFA on the side of cardiac tissue deformation [2]. Indeed, most modeling studies on RFCA reported to date have clearly neglected the influence of tissue deformation due to the insertion of the

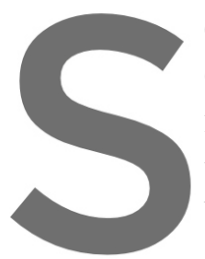
catheter. In a quest for in obtained lesion volumes for the tissue deformation the cardiac tissue and the by the circulating blood outcomes during RFCA, temperature-dependent electr
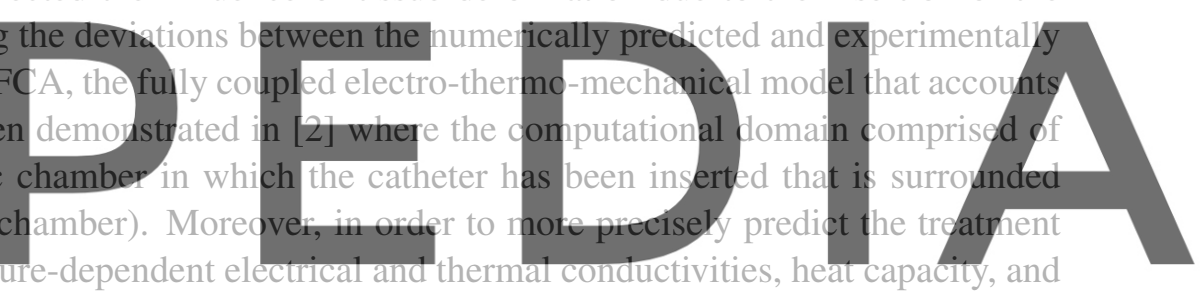

blood perfusion rate have been considered. At that point, the effect of linearly elastic and hyperelastic

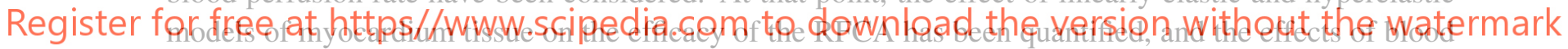

flow, the elastic modulus of myocardium tissue, and tissue-catheter contact force on the lesion volume

obtained from the fully coupled model of RFCA have been systematically investigated. From the results obtained, it was concluded that the efficacy of RFCA is significantly dependent on the electro-thermomechanical parameters and the material models of the cardiac tissue.

At the cellular scale, a novel coupled model has recently been developed to better understand the complex behaviour of biological cells subjected to piezoelectric and flexoelectric properties of their constituent organelles under the application of external forces [15]. The study took into account the nucleus, mitochondria, microtubules, cell membrane, and cytoplasm. It was revealed that flexoelectricity could be a dominant electro-elastic coupling phenomenon in biological cells, exhibiting electric fields that are four orders of magnitude higher than those generated by piezoelectric effects alone and that the mechanical degradation of the cytoskeleton results in the enhancement of both the piezo and flexoelectric responses associated with electromechanical coupling.

The important part of the consideration in the development of models for RFA of cardiac tissues represents the incorporation of fluid-structure interaction. Indeed, hemodynamics factors are critical for our 
better understanding of the cardiac function which, in its turn, should be helpful in improving diagnostics, prognosis, and therapy for a large number of pathologies [24]. Even without the RFA component, the cardiac hemodynamic modelling constitutes a challenge. It often involves the employment of special fluid-structure-interaction methods and composite approaches to muscular dynamics to simulate the complex interaction of nonlinear elastomechanics with hemodynamics. Additionally, it may include modelling components of the human circulatory system to derive the systemic pressure response, rheological models for the non-Newtonian behaviour of the blood, as well as other features, but the incorporation of electrodynamical models to include the possibility to predict the effects of arrhythmia or therapeutical ablation on the heart function has remained largely unaddressed [24]. At large, the human heart function results from a complex interplay of biochemistry, structural mechanics, and blood flow. Hence, coupled mathematical models have been a decisive player in the field of cardiovascular hemodynamics [25] and have been discussed in a number of reviews (e.g., [26]). At the same time, when it comes to RFA procedures, the area remains largely unexplored.

Following our earlier works on incorporating a number of other effects into the modelling framework for RFA, including relaxation times [1] and nonlocal effects [15], in the context of RFCA the first attempts have recently been undertaken in [27] to develop a fully-integrated coupled modelling framework which includes also non-Fourier effects and fluid-structure interaction.

Among other recent developments, we mention a fully coupled electro-thermo-mechanical model for a more accurate prediction of the treatment outcomes during RFCA, recently reported in $[6,28]$. A numerical model comprising of cardiac tissue and the cardiac chamber has been developed in which

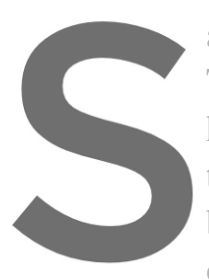
an electrode has been inserted perpendicutar to the cardiac tis\$ue to Temperature-dependent have been considered to the electrode tip on the been systematically inv of RFA is significantly dependent
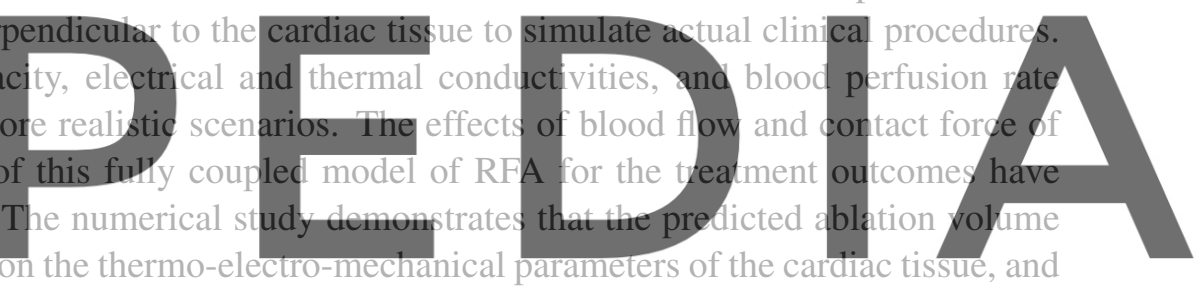
in particular on the blood flow rate in the cardiac chamber, as well as on the tissue deformation, induced

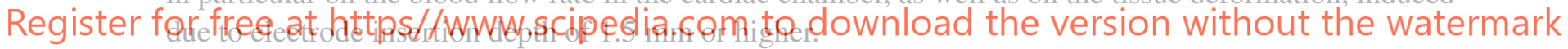

\subsection{Ablation modalities and related experimental studies}

Different modalities have been used for cardiac ablation, depending on the disease in question. Ex vivo and in vivo experiments have been carried out on lesion characteristics and tissue temperatures and the results were compared between continuous and interrupted/pulsed RFA with equal total ablation duration and contact force [29]. Such experiments have been motivated by the quest on the evaluation of the extent that ablation interruption affects lesions, given the fact that interrupted ablation is increasingly proposed as part of high-power short-duration RFA strategies and may also result from loss of contact from respiratory patterns or cardiac motion. The modelling frameworks for continuous, as well as pulsed, RFA in various medical applied contexts have been reported earlier (e.g., [13, 11] and [30], respectively). In cardiology, the pulsed-field ablation has been receiving larger attention as an ablative modality that uses short living, the strong electrical field created around the catheter to create microscopic pores in cell membranes, providing also the basis for electroporation [31]. The latter treatment technology has important implications for cardiac patients and a series of recent studies have been devoted to its various aspects, including the observed effects of electroporation pulses delivered in close vicinity of the cardiac 
pacemaker or its electrodes [32]. Moreover, recent advances in cardiac electrophysiology and related ablation procedures may lead to the development of new biomarkers for patients with heart disease [33].

\section{CONCLUSIONS}

In this paper, we have described the main features of the recently developed framework for coupled thermo-electro-mechanical modelling of ablation processes, paying special attention to cardiac ablation, as well as to the important role of domain heterogeneities which include the cell nucleus and various organelles such as microtubules. One of the directions for possible generalizations of the developed framework would be an incorporation of hybrid stochastic-deterministic components. In particular, such components would be useful for the cases described in this contribution when we further need to account for: (a) polarization of the cells (see, e.g., an interesting new model recently reported in [34]) and (b) non-equilibrium self-assembling/disassembling dynamics of microtubules. In the latter case, one of the approaches that can be pursued is based on the fact that microtubules are polymers of tubulin that form part of the cytoskeleton and are created from tubulin by spontaneous polymerization. Hence, the dynamics of microtubules can be modelled by using the ideas developed for gelation processes where hybrid models have been extensively used.

\section{ACKNOWLEDGEMENTS}

Authors are grateful to the NSERC and the CRC Program for their support. RM is also acknowledging support of the BERC 2018-2021 program and Spanish Ministry of Science, Innovation and Universities through the Agencia Es tatal de Investigacion (AEI) BCAM Severo Gchoa excellence accreditation SEY2017-0718 and the Basque Government fund AI in BCAM EXP. 2

REFERENCES

[1] Singh, S. and Melnik, R. Coupled thermo-electro-mechanica not no ological tissues and heat relaxation time effects. Physics in Medicine \& Biology (2019) 64(24):

Register for free at https//www.scipedia.com to download the version without the watermark

[2] Singh, S. and Melnik, R. Cardiac tissue deformation and the efficacy of radiofrequency ablation. Modelling the Cardiac Function Theory, Numerical Methods, Clinical Applications, 31 August - 2 September 2020, ERC, Politecnico Milano, iHEART: MCF2020.

[3] Yan, S. et al. Computer simulation study on the effect of electrode-tissue contact force on thermal lesion size in cardiac radiofrequency ablation. International Journal of Hyperthermia (2020) 37 (1): 37-48, 2020.

[4] Petras, A. et al. A computational model of open-irrigated radiofrequency catheter ablation accounting for mechanical properties of the cardiac tissue. International Journal for Numerical Methods in Biomedical Engineering (2019) 35 (11): e3232.

[5] Erdine, S. et al. . Ultrastructural changes in axons following exposure to pulsed radiofrequency fields. Pain Practice (2009) 9 (6): 407-417.

[6] Singh, S. and Melnik, R. Computational modeling of cardiac ablation incorporating electrothermomechanical interactions. ASME J of Medical Diagnostics (2020) 3 (4): 041004. 
[7] Singh, S. and Melnik, R. Thermal ablation of biological tissues in disease treatment: A review of computational models and future directions. Electromagnetic Biology and Medicine (2020) 39 (2): 49-88.

[8] Singh, S. and Melnik, R. Dynamic changes in cells exposed to pulsed radiofrequency and focus on microtubules. 9th Int Congress on Industrial and Applied Math, July 15-19, Valencia, Spain: ICIAM-2019: 435.

[9] Andrade, D. C. et al. Ablation of brainstem C1 neurons improves cardiac function in volume overload heart failure. Clinical Science (2019) 133 (3): 393-405

[10] Diaz, H. S. et al. Neuroinflammation in heart failure: new insights for an old disease. Journal of Physiology - London (2020) 598 (1): 33-59.

[11] Singh, S. and Melnik, R. Radiofrequency ablation for treating chronic pain of bones: effects of nerve locations. In: Rojas I., Valenzuela O., Rojas F., Ortuno F. (eds) Bioinformatics and Biomedical Engineering. IWBBIO 2019 (International Work-Conference on Bioinformatics and Biomedical Engineering). Lecture Notes in Computer Science, vol 11466. Springer, Cham. pp 418-429.

[12] Singh, S. and Melnik, R. Mathematical modeling of coupled electro-thermal response of nerve tissues subjected to radiofrequency fields. In: Recent Developments in Mathematical, Statistical and Computational Sciences, The V AMMCS International Conference, Waterloo, Canada, Kilgour D. M. et al (eds.) (2021) 10 pages. [13] Singh, S. and Melnik, R. Effects of heterogeneous surroundings on the efficacy of continuous ra-
diofrequency for pain relief. ICVISP 2019: Proceedings of the 3rd Intermational Conference on
Vision, Image and Signal Processing. Vancouver, Canada, August 2019, Article No.: 25, pp. 1-5,
Association for Computing Machinery, New York, USA, ISBN: 978-1-4503-7625-9.
[14] Singh, S. and Melnik, R. Domain heterogeneity in radiofrequency therapres for pain relief: a com-
putational study with coupled-models. Bioengineering (Basel, Switzertand) (2020) 7(2): 35. putational study with coupled models. Bioengineering (Basel,

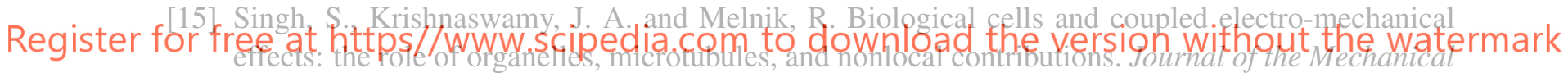 Behavior of Biomedical Materials (2020) 110: 103859.}

[16] Caporizzo, M. A. et al. Microtubules provide a viscoelastic resistance to myocyte motion. Biophysical Journal (2018) 115 (9): 1796-1807.

[17] Caporizzo, M. A. et al. Microtubules increase diastolic stiffness in failing human cardiomyocytes and myocardium. Circulation (2020) 141 (11): 902-915.

[18] Janke, C. and Magiera, M. M. The tubulin code and its role in controlling microtubule properties and functions. Nature Reviews Molecular Cell Biology (2020) 21 (6): 307-326.

[19] Caporizzo, M. A., Chen, C. Y., Prosser, B. L. Cardiac microtubules in health and heart disease. Experimental Biology and Medicine (2019) 244 (15): 1255-1272.

[20] Wenger, C. et al. Modeling TTFields application in single cells during metaphase and telophase. In: 2015 37th Annual International Conference Of The IEEE Engineering In Medicine And Biology Society $(E M B C)$ (2015) IEEE: 6892-6895. 
[21] Singh, S. and Melnik, R. Coupled Electro-mechanical Behavior of Microtubules. In: I. Rojas et al. (Eds.): IWBBIO 2020, LNBI 12108 (2020) 75-86.

[22] Singh, S. and Melnik, R. Microtubule Biomechanics and the Effect of Degradation of Elastic Moduli. In: V. V. Krzhizhanovskaya et al. (Eds.): ICCS 2020, LNCS 12142 (2020) 348-358.

[23] Ruiz-Baier, R. et al. Modelling thermo-electro-mechanical effects in orthotropic cardiac tissue. Commun. Comput. Phys. (2020) 27: 87-115.

[24] Schenkel, T. et al. The Karlsruhe heart model KaHMo: a modular framework for numerical simulation of cardiac hemodynamics. In: :Dossel, O. and Schlegel, W. C. (eds.), 11th International Congress of the IUPESM/World Congress on Medical Physics and Biomedical Engineering, Munich, September 7-12, 2009, IFMBE Proceedings, Vol 25, Pt 4: Image Processing, Biosignal Processing, Modelling and Simulation, Biomechanics (2010) 615-618.

[25] Muehlhausen, M. P., Janoske, U., Oertel, H. Implicit partitioned cardiovascular fluid-structure interaction of the heart cycle using non-Newtonian fluid properties and orthotropic material behavior. Cardiovascular Engineering and Technology (2015) 6 (1): 8-18.

[26] Doost, S. N. et al. Heart blood flow simulation: a perspective review. Biomedical Engineering Online (2016) 15: 101.

[27] Singh, S. and Melnik, R. Fluid-structure interaction and non-Fourier effects in coupled electrothermo-mechanical models for cardiac ablation. (2021) Submitted.

[28] Singh, S. and Melnik, R. Computtional model of radiofrequency ablation of cardiac tissues incorporating thermo-electro-mechanical interactions. Proceedings of the ASME 2020: International Mechanical Engineering Congress and Exposition IMECE2020, November 16-19, 2020, Portland, OR, USA, Paper No: IMECE2020-23367, 8 pages.

[29] Rogers, A. J. et al. Continuous ablation improves lesion maturation compared with intermittent ablation strategies. Journal of Cardiovascular Electrophysiology (2020) 31 (7): 1687-1693.

[30] Singh, S. and Melnik, R. Computational Analysis of Pulsed Radiofrequency Ablation in Treating Chronic Pain. In: Computational Science - ICCS 2019, Pt IV, Rodrigues, J. M. F. et al (eds.), Lecture Notes in Computer Science (2019) Vol. 11539: 436-450.

[31] Anic, A., Breskovic, T., Sikiric, I. Pulsed field ablation: a promise that came true. Current opinion in cardiology (2021) 36 (1): 5-9.

[32] Jarm, T. et al. Investigation of safety for electrochemotherapy and irreversible electroporation ablation therapies in patients with cardiac pacemakers. Biomedical Engineering Online (2020) 19 (1): 85.

[33] Kapa, S. et al. Year in review in cardiac electrophysiology. Circulation - Arrhythmia and Electrophysiology (2020) 13 (6): CIRCEP.120.008733.

[34] Copos, C. and Mogilner, A. A hybrid stochastic-deterministic mechanochemical model of cell polarization. Molecular Biology of the Cell (2020) 31 (15): 1637-1649. 